Does video game-based balance-training improve gait stability in children with cerebral palsy?

Peer-reviewed author version

MEYNS, Pieter; Bras, Chloé; Harlaar, Jaap; van de Pol, Laura; Barkhof, Frederik \& Buizer, Annemieke (2018) Does video game-based balance-training improve gait stability in children with cerebral palsy?. In: Gait \& posture, 65(S1), p. 105-106.

DOI: 10.1016/j.gaitpost.2018.06.078

Handle: http://hdl.handle.net/1942/26653 
Title: Does video game-based balance-training improve gait stability in children with cerebral palsy?

Authors:

Pieter Meyns, Chloé Bras, Jaap Harlaar, Laura van de Pol, Frederik Barkhof, Annemieke Buizer

Introduction:

Video games are increasingly used in balance rehabilitation since literature has indicated promising effects on motivation[1] and functional outcomes[2] in several patient populations. Several pilot studies investigated the effectiveness of video game-based balance-training (VGBT) in children with cerebral palsy $(\mathrm{CPc})[3,4]$, as these children experience poor balance control during standing and walking due to sensorimotor disorders resulting from non-progressive brain lesions[5]. These studies, however, yielded inconsistent results. This could be caused by the clinical balance scales used, which might be insensitive to training-induced improvements, as these scales are often ordinal-scale and composite scores of walking and standing balance[6].

\section{Research Question:}

Is VGBT effective to improve three balance outcomes in CPc; Pediatric Balance Scale (PBS; frequently used clinical ordinal-scale measure of functional balance), margins of stability (MoS; ratio-scale gait stability measure based on whole-body biomechanics) and MoS variability (sdMoS)?

Methods:

Preliminary data of our registered trial (NTR6034) is presented. Eight CPc (8-16 years old) were included (table 1), if they had: bilateral spastic CP, GMFCS-level 2, no surgery $<12$ months, and no Botulinum-Toxin A injections $<6$ months. VGBT comprised 6 weeks home-based X-box One Kinect (Microsoft) training. Kinect sports games focused on balance (tennis, football, bowling) were used $5 x /$ week, 30min/session. Time played was monitored. Pre and post-VGBT, participants performed unperturbed treadmill walking trials at self-selected speed. Total body (Plug-In-Gait) kinematics were collected via a 10-camera Vicon motion-capture system at $120 \mathrm{~Hz}$. Medio-lateral MoS was calculated as the position of the extrapolated center of mass (XCOM) relative to the lateral malleolus of the leading foot[7]. XCoM was defined as CoM, plus its velocity times a factor: $\mathrm{V}$ (CoM height/acceleration of gravity). High MoS indicates a large distance between XCoM and lateral border of base of support, and represents stable gait. Variability of MoS (sdMoS) was determined (high variability indicates less stable gait). Functional balance was assessed with PBS; a 14-item balance measure concerning everyday tasks (on 56 points; high PBS indicates good balance).

Results:

Only sdMoS improved significantly post-VGBT (table1). 
Title: Does video game-based balance-training improve gait stability in children with cerebral palsy?

\begin{tabular}{|c|c|c|c|c|c|c|c|c|c|c|c|c|}
\hline Table1 & CPi01 & CPi02 & CPi03 & CPi04 & CPi05 & CPi06 & CPi07 & CPi08 & Mean & SD & $\begin{array}{c}\text { Wilcoxon Signed } \\
\text { Rank Z test }\end{array}$ & $p$-value \\
\hline gender & male & male & male & male & male & female & male & male & & & & \\
\hline age (yrs) & 10.6 & 9.8 & 11.9 & 8.1 & 10.6 & 15.8 & 14.3 & 13.6 & 11.8 & 2.6 & & \\
\hline pre-VGBT & 109 & 69 & 72 & 43 & 58 & 42 & 48 & 44 & & & & \\
\hline Pre-VGBT & 14.6 & 17.5 & 20.3 & 17.1 & 15.1 & 22.8 & 15.9 & 18.6 & 17.8 & 2.8 & & \\
\hline Post-VGBT & 13.3 & 16.9 & 21.4 & 17.0 & 17.6 & 27.0 & 15.0 & 19.6 & 18.5 & 4.3 & 24.00 & 0.401 \\
\hline \multicolumn{13}{|l|}{ sdMoS (cm) } \\
\hline \multicolumn{13}{|l|}{ PBS } \\
\hline Pre-VGBT & 41 & 51 & 54 & 51 & 50 & 49 & 56 & 41 & 49.1 & 5.4 & & \\
\hline Post-VGBT & 46 & 49 & 55 & 50 & 48 & 49 & 56 & 52 & 50.6 & 3.5 & 12.50 & 0.673 \\
\hline
\end{tabular}

Discussion:

Preliminary results indicate that sdMoS is most sensitive to assess VGBT-induced balance improvements in CPc. This finding suggests a transfer from standing balance-training towards gait stability. Contrarily, VGBT did not induce improvements on PBS, which may indicate that PBS is less sensitive to training-induced changes or that training-induced sdMoS improvements are not clinically relevant. Half of the children increased (decreased) MoS after VGBT; i.e. the distance increased (decreased) between XCoM and lateral border of the base of support. These findings need further investigation taking into account whether individual patients showed increased step widths as compensation to decrease gait instability.

Acknowledgments:

PM was Marie-Skłodowska Curie-fellow (EU; n660458).

References:

[1]Meyns, Dev.Neurorehabil-2017;9:1-20, [2]Tatla, Dev.Med.Child.Neurol-2013;55:593-601, [3]Jelsma, Dev.Neurorehabil-2013;16:27-37, [4]Brien, Pediatr.Phys.Ther-2011;23:258-66, [5]Bruijn, Res.Dev.Disabil-2013;34:1689-99, [6]Meyns, Gait.Posture-2017;57:234-235, [7]Hak, PLoS.One2013;8:e82842 